\title{
ATM Layouts with Bounded Hop Count and Congestion ${ }^{\star}$
}

\author{
Michele Flammini ${ }^{1,2}$, Enrico Nardelli ${ }^{1,3}$ and Guido Proietti ${ }^{1}$ \\ 1 Dipartimento di Matematica Pura ed Applicata, Università degli Studi di L'Aquila, \\ via Vetoio loc.Coppito, I-67100 L'Aquila, Italy. \\ E-mail: \{flammini,nardelli,proietti\}@univaq.it \\ 2 Project SLOOP I3S-CNRS/INRIA/Université de Nice-Sophia Antipolis, \\ 930 route des Colles, F-06903 Sophia Antipolis Cedex, France \\ ${ }^{3}$ Istituto di Analisi dei Sistemi ed Informatica, Consiglio Nazionale delle Ricerche \\ Viale Manzoni 30, I-00185 Roma, Italy
}

\begin{abstract}
In this paper we consider two new cost measures related to the communication overhead and the space requirements associated to virtual path layouts in ATM networks, that is the edge congestion and the node congestion. Informally, the edge congestion of a given edge $e$ at an incident node $u$ is defined as the number of VPs terminating or starting from $e$ at $u$, while the node congestion of a node $v$ is defined as the number of VPs having $v$ as an endpoint. We investigate the problem of constructing virtual path layouts allowing to connect a specified root node to all the others in at most $h$ hops and with maximum edge or node congestion $c$, for two given integers $h$ and $c$. We first give tight results concerning the time complexity of the construction of such layouts for both the two congestion measures, that is we exactly determine all the tractable and intractable cases. Then, we provide some combinatorial bounds for arbitrary networks, together with optimal layouts for specific topologies such as chains, rings, grids and tori. Extensions to $d$-dimensional grids and tori with $d>2$ are also discussed.
\end{abstract}

\section{Introduction}

The Asynchronous Transfer Mode (ATM for short) is the most popular networking paradigm for Broadband ISDN [11, 10, 13]. It transfers data in the form of small fixed-size cells, that are routed independently according to two routing fields at their header: the virtual channel index (VCI) and the virtual path index (VPI). At each intermediate switch, these fields serve as indices to two routing tables, and the routing is done in accordance to the predetermined information in the appropriate entries.

\footnotetext{
* Work supported by the EU TMR Research Training Grant N. ERBFMBICT960861, by the EU ESPRIT Long Term Research Project ALCOM-IT under contract N. 20244 and by the Italian MURST $40 \%$ project "Algoritmi, Modelli di Calcolo e Strutture Informative".
} 
Routing in ATM is hierarchical in the sense that the VCI of a cell is ignored as long as its VPI is not null. Thus, given the graph underlying the network, it is possible to distinguish between the virtual paths, VPs for short, that are based on VPIs and are constituted by a sequence of successive edges or physical links, and virtual channels or VCs, that correspond to VCIs and are obtained by concatenating a given subset of VPs.

The VP layouts determined by the VPIs and VCIs entries can be evaluated with respect to different cost measures. In particular, a fundamental parameter is the hop count, which is given by the number of VPs which comprise the path of a VC and determines the efficiency of the setup of the VC (see, e.g., $[2,14,15])$. Another cost measure widely considered in the literature is the load of a physical edge, which is given by the number of virtual paths that share the edge. This number determines the size of the VP routing tables (see, e.g., [4]). Finally, the stretch factor is the ratio between the length of the path that a VC takes in the physical graph and the shortest possible path between its endpoints. This parameter controls the efficiency of the utilization of the network. For further details and technical justifications of the model for ATM networks see for instance $[1,9]$.

Some empirical results on the ATM layout problem have been given in $[1,12]$ and some more theoretical analysis in $[9,3,7,8,5]$.

In particular, in [9] the computational complexity of determining the existence of a VP layout for a given network within a given maximum hop count and a given maximum load has been investigated and the authors have shown that this problem is NP-complete when there is no limit on the stretch factor. In [9] also some polynomial construction algorithms have been given for trees for the stretch factor equal to one, i.e. when the physical routed paths are shortest. These results have been extended in [5], where an exact characterization of the tractable and intractable cases has been given for shortest path layouts.

Concerning layout constructions for specific topologies, optimal and nearly optimal results for chains and trees have been provided in $[9,3,8]$, while to the best of our knowledge no optimal results are known for other topologies like grids and tori.

In this paper we consider two new cost measures associated to virtual path layouts: the edge congestion, which is given by the number of VPs terminating or starting from a given edge at a given node, and the node congestion, that is the number of VPs having as an endpoint a given node. These cost measures take into account in a balanced (in case of edge congestion) or global (for node congestion) way the communication overhead at every given node. In fact, they are indicative of the number of accesses in the VC tables, as such tables are readed at the end of the VPs. Moreover, these parameters influence directly the dimension of VC tables, as any VP which increases the congestion of an edge or of a node causes a number of entries in the corresponding VC table equal to the number of VCs such a VP belongs to.

As in [9] and [5], we will focus on layouts that enable the routing between all nodes and a single root node (rather than between any pair of nodes), under the 
assumption of a stretch factor equal to one, that is all the physical routed paths are the shortest. In fact, this restricted case can be seen as a building block for more complex routing problems and nevertheless its simplicity it has not been fully understood yet.

After showing some general combinatorial results holding for any network, we give tight results on the time complexity of constructing optimal rooted virtual path layouts. In fact, for the edge congestion case, we exactly establish the border between tractability and intractability, by determining the lowest (constant) values of $h$ and $c$ that make the problem computationally hard. Moreover, we give efficient construction algorithms for all the tractable cases. Analogous results are obtained for the node congestion case. We then provide some optimal layouts for specific networks, such as chains, rings, grids and tori. Extensions to $d$ dimensional grids and tori with $d>2$ are also discussed.

The paper is organized as follows: In Section 2 we define the preliminary notation and definitions. In Section 3 we give some basic results for the considered cost measures. In Section 4 we provide the above-mentioned time complexity results. In Section 5 we present the optimal layouts for specific topologies and finally, in Section 6, we give some concluding remarks and list some open problems.

\section{Preliminaries}

We model the network as an undirected graph $G=(V, E)$, where nodes in $V$ represent switches and edges in $E$ are the point-to-point physical communication links.

Definition 1. A rooted virtual path layout (or simply layout) $\Psi$ is a collection of paths in $G$, termed virtual paths (VPs for short), and a node $r \in V$, termed the root of the layout.

Definition 2. The hop count $\mathcal{H}(v)$ of a node $v \in V$ in a layout $\Psi$ is the minimum number of VPs whose concatenation forms a shortest path in $G$ from $v$ to $r$. If no such VPs exist, define $\mathcal{H}(v) \equiv \infty$.

Definition 3. The maximal hop count of a layout $\Psi$ is $\mathcal{H}_{\max }(\Psi) \equiv$ $\max _{v \in V}\{\mathcal{H}(v)\}$.

Given $v \in V$, let us denote as $I(v)$ the set of the edges in $E$ incident to $v$.

Definition 4. Given $v \in V$ and $e \in I(v)$, the edge congestion $\mathcal{E}(e, v)$ of the edge $e$ with respect to $v$ in a layout $\Psi$ is the number of VPs $\psi \in \Psi$ that include $e$ and have $v$ as an endpoint.

Definition 5. The maximal edge congestion $\mathcal{E}_{\max }(\Psi)$ of a layout $\Psi$ is $\max _{v \in V, e \in I(v)} \mathcal{E}(e, v)$. 
A layout $\Psi$ with $\mathcal{H}_{\max }(\Psi) \leq h$ and $\mathcal{E}_{\max }(\Psi) \leq c$ is called a $(h, c\rangle$-edge layout.

At each node of the network, a more global congestion measure can be considered which takes into account the total cost required at the node.

Definition 6. Given $v \in V$, the node congestion $\mathcal{N}(v)$ of $v$ in a layout $\Psi$ is the number of VPs $\psi \in \Psi$ such that $v$ is an endpoint of $\psi$.

Definition 7. The maximal node congestion $\mathcal{N}_{\max }(\Psi)$ of a layout $\Psi$ is $\max _{v \in V} \mathcal{N}(v)$.

A layout $\Psi$ with $\mathcal{H}_{\max }(\Psi) \leq h$ and $\mathcal{N}_{\max }(\Psi) \leq c$ is called a $\langle h, c\rangle$-node layout.

Clearly, the hop count and the edge (or node) congestion are conflicting parameters, as in general a low hop count requires an high congestion and a low congestion causes a high hop count. Thus, a very natural problem arises in which one parameter is traded for the other. Moreover, once fixed two bounds $h$ and $c$ respectively on the hop count and on the edge (or node) congestion, in a parametric family of graphs it makes sense to consider the problem of determining the highest order graph that admits a layout respecting such bounds.

Definition 8. Let $\mathcal{G}$ be a family of graphs $\mathcal{G}$. For any two positive integers $h$ and $c, E_{\mathcal{G}}(h, c)$ (resp. $N_{\mathcal{G}}(h, c)$ ) is defined as the maximum integer $N$ such there exist an $N$-node graph in $\mathcal{G}$ with a $\langle h, c\rangle$-edge layout (resp. a $\langle h, c\rangle$-node layout).

For the sake of brevity, when clear from the context, we will denote $E_{\mathcal{G}}(h, c)$ and $N_{\mathcal{G}}(h, c)$ respectively as $E(h, c)$ and $N(h, c)$.

Notice that all the definitions above assume a stretch factor equal to one, i.e. all the physical routed paths are the shortest.

\section{Basic results}

In this section we give some basic results related to the new cost measures considered in the paper.

First of all, let us establish upper bounds on the number of nodes in graphs admitting $\langle h, c\rangle$-edge layouts or $\langle h, c\rangle$-node layouts.

Given a graph $G$ with a specified root node $r$, we say that a non root node $u$ has parameter $d$ if it has exactly $d$ edges $\left\{u, v_{1}\right\}, \ldots,\left\{u, v_{d}\right\}$ such that for each $i$, $1 \leq i \leq d, u$ is on a shortest path from $r$ to $v_{i}$. Informally, in the edge congestion case, the parameter of a node expresses its ability to reach other nodes, as it is equal to the number of edges that can be used to construct new VPs in any shortest path layout. Let the parameter of a family of graphs $\mathcal{G}$ be the maximum parameter of a non root node of a graph in $\mathcal{G}$.

Lemma9. Let $\mathcal{G}$ a family of the graphs with parameter $d$ and such that every root $r$ has degree at most $d_{r}$. Then $E(h, c) \leq 1+c d_{r} \frac{(c d)^{h}-1}{c d-1}$. 
Proof. The lemma can be proved by observing that from the root node $r$ in one hop it is possible to reach at most $c \cdot d_{r}$ nodes; each node with hop count 1 can then reach in another hop at most $c d$ other nodes (for a total of $c d_{r}(c d)$ nodes), and this holds for every node with hop count at least 1 , since the physical routed paths have to be the shortest and thus each node can use at most $d$ outgoing edges to reach other nodes. Hence,

$$
\begin{gathered}
E(h, c) \leq 1+c d_{r}+c d_{r}(c d)+c d_{r}(c d)^{2}+\ldots+c d_{r}(c d)^{h-1}= \\
1+c d_{r} \sum_{i=0}^{h-1}(c d)^{i}=1+c d_{r} \frac{(c d)^{h}-1}{c d-1} .
\end{gathered}
$$

Lemma 10. For any family of graphs $\mathcal{G}, N(h, c) \leq \frac{c(c-1)^{h}-2}{c-2}$.

Proof. Starting from the root node $r$, in one hop it is possible to reach at most $c$ nodes; each node with hop count 1 can then reach in another hop at most $c-1$ other nodes, and this holds for every node with hop count at least 1 , since the VP through which a node is reached contributes 1 to its node congestion. Thus,

$$
\begin{gathered}
N(h, c) \leq 1+c+c(c-1)+c(c-1)^{2}+\ldots+c(c-1)^{h-1}= \\
1+c \sum_{i=0}^{h-1}(c-1)^{i}=1+c \frac{(c-1)^{h}-1}{c-2}=\frac{c(c-1)^{h}-2}{c-2} .
\end{gathered}
$$

We finally point out that in this context it does not make sense to consider layouts with unbounded physical routed lengths. In fact, optimal layout constructions for the node congestion case can be determined as follows. Consider any ordering of the nodes, except the root. Then, the root reaches through a VP in one hop the first $c$ nodes, and iteratively in the order each reached node is assigned a VP to all the next $(c-1)$ unreached nodes, thus attaining the upper bound of Lemma 10, which holds also under the assumption of an unbounded stretch factor. In the edge congestion case the construction is slightly more complicated, since nodes have to be ordered non increasingly with respect to their degrees. Notice that, since to the purpose of minimizing the edge congestion VPs have not necessarily to correspond to simple physical paths, at every node the incident VPs can be equally distributed among its incident edges. Thus an optimal layout can be easily determined.

Optimal layouts can be easily found even in the gossip case in which, by respecting the bounds on the edge or node congestion, each node wants to reach every other node in at most a given number of hops. Here the construction becomes a pure combinatorial graph design problem. In fact, if the node congestion is bounded by $c$, there is a layout for a graph $G$ within a given hop count $h$ if and only if there exists a $c$-bounded degree graph with diameter $h$ and at least the same number of nodes of $G$. Any embedding of such a graph on $G$ gives the 
desired layout. A similar argument holds for the edge congestion, but here there is a layout respecting $h$ and $c$ if there exists a graph with the same number of nodes, diameter $h$ and such that, if we denote as $d_{i}$ the degree of node $i$ in the initial graph $G$, the $i$-th node of the graph has degree at most $c \cdot d_{i}$.

\section{Time complexity results}

In this section we prove tight time complexity results for the problem of the construction of optimal layouts, by giving an exact characterization of all the tractable and intractable case. Some of the proofs are omitted in this extended abstract and will appear in the full version of the paper.

According to the previous sections, the following decision problem naturally arises.

Definition 11. $\langle h, c\rangle$-edge layout Problem:

INSTANCE: A network $G=(V, E)$ and a given root $r \in V$.

QUESTION: Is there a $\langle h, c\rangle$-edge layout for $G$ with root $r$ ?

By using proof techniques like those in [5], surprisingly a very similar result holds for the edge congestion case.

Theorem 12. The $\langle h, c\rangle$-edge layout problem is NP-complete for any $h$ and $c$, except for the cases $h=1$, any $c$ and $h=2, c=1$.

Suitable flow constructions prove the following theorem.

Theorem 13. The $\langle h, c\rangle$-edge layout problem is polynomially solvable if $h=1$ (any c) or $h=2, c=1$.

Different results instead hold for the node congestion case. In fact, once fixed $h$ and $c$, the problem of determining the existence of an $\langle h, c\rangle$-node layout for any graph $G$ has a polynomial-time complexity, since from Lemma 10 we know that the number of nodes in $G$ is $N(h, c) \leq \frac{c(c-1)^{h}-2}{c-2}$, i.e. it is always bounded by a constant. Hence, it makes sense to consider different decision problems in which $h$ or $c$ are not constant, that is are part of the input of the instance.

Definition 14. $(h$, 미-node layout Problem:

INSTANCE: A network $G=(V, E)$, a given root $r \in V$ and a positive integer $c$.

QUESTION: Is there a $\langle h, c\rangle$-node layout for $G$ with root $r$ ? 


\section{Definition 15. $\langle\square, c\rangle$-node layout Problem:}

INSTANCE: A network $G=(V, E)$, a given root $r \in V$ and a positive integer $h$.

QUESTION: Is there a $(h, c)$-node layout for $G$ with root $r$ ?

The following results can then be proved.

Theorem 16. For $h \geq 2$ the $\langle h, \square\rangle$-node layout problem is NP-complete.

Proof. We first show that the $(2$, D)-node layout problem is NP-complete by providing a polynomial time transformation from the Dominating Set problem (DS) (known to be NP-complete; see [6]). In order to complete the proof, we then prove that if the $\langle h, \square\rangle$-node layout problem is NP-complete, then also the $\langle h+1, \square\rangle$-node layout problem is NP-complete.

In the DS problem we have a universe set $U=\left\{u_{1}, \ldots, u_{m}\right\}$ of $m$ elements, a family $\left\{A_{1}, \ldots, A_{f}\right\}$ of $f$ subsets of $U$ and an integer $k \leq f$; we want to decide if there exist $k$ subsets $A_{j_{1}}, \ldots, A_{j_{k}}$ which cover $U$, i.e. such that $\bigcup_{i=1}^{k} A_{j_{i}}=U$.

Starting from an instance $I_{D S}$ of DS, we construct a graph $G$ that admits a $\langle 2, c\rangle$-node layout with $c=m$ if and only $I_{D S}$ admits a cover.

Let $G=(V, E)$, where $V=\{r\} \cup V_{1} \cup V_{2} \cup V_{3} \cup V_{4}$ and $E=E_{1} \cup E_{2} \cup E_{3} \cup E_{4}$, with:

$$
\begin{aligned}
& V_{1}=\left\{v_{a} \mid a=1, \ldots, m-k\right\}, \\
& V_{2}=\left\{q_{b} \mid b=1, \ldots, m-(f-k)-1\right\}, \\
& V_{3}=\left\{w_{c} \mid c=1, \ldots, f\right\}, \\
& V_{4}=\left\{z_{d} \mid d=1, \ldots, m\right\}, \\
& \quad \text { and } \\
& E_{1}=\left\{\left\{r, v_{a}\right\} \mid a=1, \ldots, m-k\right\}, \\
& E_{2}=\left\{\left\{v_{1}, q_{b}\right\} \mid b=1, \ldots, m-(f-k)-1\right\}, \\
& E_{3}=\left\{\left\{v_{1}, w_{c}\right\} \mid c=1, \ldots, f\right\}, \\
& E_{4}=\left\{\left\{w_{c}, z_{d}\right\} \mid u_{d} \in A_{c}\right\} .
\end{aligned}
$$

Informally, in the reduction graph each subset $A_{c}$ corresponds to the subgraph induced by node $w_{c}$ and all nodes $z_{d}$ such that $u_{d} \in A_{c}$, which are all connected to $w_{c}$. The idea underlying our construction is that since at most $k$ of the nodes $w_{c}$ can be reached from $r$ in one hop, if there are $k$ dominating sets in $I_{D S}$, then all nodes of $G$ can be reached from $r$ in at most 2 hops.

Assume there are $k$ dominating sets $A_{j_{1}}, \ldots, A_{j_{k}}$. We show that there exists a $\langle 2, c\rangle$-node layout for $G$. The VPs of $\Psi$ are constituted by all edges in $E_{1} \cup E_{2} \cup E_{3}$, the edges $\left\{v_{1}, w_{c}\right\} \in E_{3}$ such that $A_{c}$ is not one of the dominating sets, i.e. $c \neq j_{i}, i=1, \ldots, k$, and finally the VPs $\left\langle r, v_{1}, w_{j_{i}}\right)$ for $i=1, \ldots, k$ (which correspond to the $k$ dominating sets). By construction, $\mathcal{N}(v) \leq m=c$ for each node $v \in E$. In order to check that $\mathcal{H}_{\max }(\Psi) \leq 2$, it suffices to observe that all nodes $v_{a} \in V_{1}$ are reached in one hop, nodes $q_{b} \in V_{2}$ are reached in two hops, nodes $w_{c} \in V_{3}$ not corresponding to dominating sets are reached in two hops, nodes $w_{j_{i}} \in V_{2}$ corresponding to dominating sets are reached in one hop (through the VP $\left\langle r, v_{1}, w_{j_{i}}\right\rangle$ ), and as nodes $w_{j_{1}}, \ldots, w_{j_{k}}$ correspond to the $k$ 
dominating sets, all nodes $z_{d} \in V_{4}$ are reached in two hops, since every one of them is connected to at least one $w_{j_{i}}$.

It remains to show that if there are no $k$ dominating sets, then no $\langle 2, c\rangle$-node layout $\Psi$ for $G$ exists. Consider any layout $\Psi$ for $G$. Notice first that each of the edges $\left\{r, v_{a}\right\}$ must belong to $\Psi$, otherwise $\mathcal{H}\left(v_{a}\right)=\infty$. Similarly, since each node $q_{b}$ must be reached through a shortest path, either the edge $\left\{v_{1}, q_{b}\right\}$ or the path $\left\langle r, v_{1}, q_{b}\right\rangle$ must be a VP of $\Psi$. Without loss of generality we can assume that the first case holds, as otherwise inserting $\left\{v_{1}, q_{b}\right\}$ in the set of the VPs of $\Psi$ and replacing another VP starting from $v_{1}$ with a longer one directly from $r$, $\mathcal{H}\left(q_{b}\right)=2$ and the hop count and node congestion of all the other nodes can only be decreased. Then, there are $f$ nodes $w_{c} \in V_{3}$ to be reached along shortest paths and this can be done only through the remaining $f$ VPs, of which $k$ can start from the root and $f-k$ from $v_{1}$, yielding respectively hop count 1 and 2. Hence, no node in $V_{4}$ can be reached in two hops without exploiting a VP starting from a node $w_{c} \in V_{3}$. Let $w_{j_{1}}, \ldots, w_{j_{k}}$ be the $k$ nodes in $V_{3}$ such that $\mathcal{H}\left(w_{j_{i}}\right)=1, i=1, \ldots, k$. Since there are no $k$ dominating sets, then at least one node $z_{c}$ is not connected to any of the nodes $w_{j_{1}}, \ldots, w_{j_{k}}$, and therefore $\mathcal{H}\left(z_{c}\right) \geq 3$.

In order to complete the proof, we now show that if the $\langle h, \square\rangle$-node layout problem is NP-complete, then also the $\langle h+1, \square\rangle$-node layout problem is NPcomplete.

This can be accomplished simply by observing that given the graph $\delta=$ $(V, E)$ and the node congestion $c$ corresponding to the instance of the $\langle h, \square\rangle$ node layout problem, the graph $G^{\prime}$ obtained by adding a new root node $r^{\prime}$ and connecting it to the old root $r$ of $G$ and to $c-1$ additional new nodes has a $\langle h+1, c\rangle$-node layout if and only if $G$ has a $\langle h, c\rangle$-node layout. In fact, each of the new inserted edges in $G^{\prime}$ has to be a $V P$, so $\mathcal{H}(r)=1$, and all the remaining VPs car only start from the old root $r$.

A graph $G=(V, E)$ admits a $\langle 1, c\rangle$-node layout if and only if $c \geq|V|-1$, as from the root it is possible to reach with a VP at most $c$ nodes and $c$ nodes can always be reached along shortest paths by means of $c$ VPs leaving the root. Thus the following theorem is trivially proved.

Theorem 17. The $\langle 1, \square\rangle$-node layout problem is solvable in polynomial time.

Let us now turn our attention to the second decision problem associated to the node congestion case, where $c$ is a fixed constant and the hop count $h$ belongs to the input of the instance.

Theorem 18. For $c \geq 3$ the $(\square, c)$-node layout problem is NP-complete.

Theorem 19. For $c \leq 2$ the $\langle\square, c\rangle$-node layout problem is solvable in polynomial time.

All the polynomial time algorithms for the above tractable cases are constructive, that is they either find the proper layout (if it exists) or they return a negative answer. 
A final interesting remark concerns the $\langle h, c\rangle$-edge layout problem. In fact, the same argument which leaded to the introduction of two different problems in the node congestion case, i.e. the constant upper bound on the total number of nodes in the graph, proves the following theorem.

Theorem 20. Given any positive integer $\delta$, the restriction of the $\langle h, c\rangle$-edge layout problem to the class of the graphs with maximum node degree $\delta$ is solvable in polynomial time.

\section{$5 \quad$ Results for specific topologies}

In this section we give optimum layouts for specific topologies.

Let us consider first a chain or path of nodes with node set $V=\{1, \ldots N\}$ and edge set $E=\{\{i, i+1\} \mid 1 \leq i<N\}$. In order to give worst case estimations on the longest chain admitting a $\langle h, c\rangle$-edge or $\langle h, c\rangle$-node layout, we assume $r=1$ as the root node.

Theorem 21. Let $\mathcal{P}$ be the family of chain (or path) graphs. Then $E(h, c)=\frac{c^{h+1}-1}{c-1}$ and $N(h, c)=\frac{c(c-1)^{h}-2}{c-2}$

Proof. By Lemma $9 E(h, c) \leq 1+c \frac{c^{h}-1}{c-1}=\frac{c^{h+1}-1}{c-1}$ and by Lemma 10 $N(h, c) \leq \frac{c(c-1)^{h}-2}{c-2}$.

The lower bound on $E(h, c)$ (resp. $N(h, c)$ ) follows by observing that from the root of any chain it is possible to reach the next $c$ nodes in one hop, and from each node with hop count at least one again the first next unreached $c$ nodes (resp. $c-1$ nodes), thus yielding $E(h, c) \geq 1+c+c^{2}+\ldots+c^{h}=\frac{c^{h+1}-1}{c-1}$ and $N(h, c) \geq 1+c+c(c-1)+\ldots+c(c-1)^{h-1}=\frac{c(c-1)^{h}-2}{c-2}$.

A ring graph consists of a node set $V=\{0, \ldots, N-1\}$ and an edge set $E=\left\{\left\{i,(i+1)_{\bmod N}\right\} \mid 1 \leq i<N\right\}$. As a ring is node-symmetric, without loss of generality it is possible to choose any node as the root. By arguments similar those ones for chain graphs it is possible to prove the following theorem.

Theorem 22. Let $\mathcal{R}$ be the family of ring graphs, then $E(h, c)=2 \frac{c^{h+1}-1}{c-1}-1$ and $N(h, c)=\frac{c(c-1)^{h}-2}{c-2}$ if $c$ is even, otherwise $N(h, c)=1+\frac{(c-1)^{h+1}-1}{c-2}$.

We now turn our attention to the 2-dimensional extensions of chains and rings, that is to grids and tori.

Given a square grid $G_{n \times n}$ of $N=n^{2}$ nodes, with node set $V=\{(i, j) \mid 1 \leq$ $i \leq n, 1 \leq j \leq n\}$ and edge set $E=\{\{(i, j),(i+1, j)\} \mid 1 \leq i<n, 1 \leq j \leq$ $n\} \cup\{\{(i, j),(i, j+1)\} \mid 1 \leq i \leq n, 1 \leq j<n\}$, again in order to give worst case estimations on the largest grid admitting a $\langle h, c\rangle$-edge or $\langle h, c\rangle$-node layout, we assume $r=(1,1)$ as the root node.

For the case of edge congestion $c=1$, as stated by the following theorem the dimension of the largest grid admitting a $\langle h, c\rangle$-edge layout is dominated by the maximum number of nodes reachable in $h$ hops along the first row or column. 
Theorem 23. Let $\mathcal{P}^{2}$ be the family of square grid graphs, then $E(h, 1)=h^{2}$ if $h \leq 3$, otherwise $E(h, 1)=(h+1)^{2}$.

Sketch of proof. By Lemma 9 , taking $d_{r}=2$ and $d=2$, the maximum number of nodes reachable in $h$ hops is $N \leq \frac{(2 c)^{h+1}-1}{2 c-1}=2^{h+1}-1$, which gives $E(h, 1) \leq$ $h^{2}$ for $h \leq 3$. Moreover, the maximum number of nodes along the first row or column reachable from the root in $h$ hops is $h+1$ (the root included), thus yielding $E(h, 1) \leq(h+1)^{2}$ for $h>3$. The layout attaining such bounds will be shown in the full paper.

Theorem 24. Let $\mathcal{P}^{2}$ be the family of square grid graphs. Then, for $c \geq 4$, $E(h, c)=\left\lfloor\sqrt{N_{h, c}}\right\rfloor^{2}$, where $N_{h, c}=\frac{(2 c)^{h+1}-1}{2 c-1}$.

Proof. Again by Lemma 9 with $d_{r}=2$ and $d=2$, the maximum number of nodes reachable in $h$ hops is $N_{h, c} \leq \frac{(2 c)^{h+1}-1}{2 c-1}$. Since every grid has a quadratic number of nodes, that is $n^{2}$ for a given integer $n \geq 1$, the upper bound on $E(h, c)$ derives directly by observing that $n=\left\lfloor\sqrt{N_{h, c}}\right\rfloor$ is the maximum integer such that $n^{2} \leq N_{h, c}$.

In order to provide an optimal layout, given a square grid $G$ with at least $N_{h, c}$ nodes, we define a gridoid $G_{h}$ as the subgrid of $G$ induced by nodes $(i, j)$ with $i \leq$ $\left\lfloor\sqrt{N_{h, c}}\right\rfloor$ and $j \leq\left\lfloor\sqrt{N_{h, c}}\right\rfloor$, i.e. the $\left\lfloor\sqrt{N_{h, c}}\right\rfloor \times\left\lfloor\sqrt{N_{h, c}}\right\rfloor$ subgrid induced by the first $\left\lfloor\sqrt{N_{h, c}}\right\rfloor$ rows and columns, plus the $N_{h, c}-\left\lfloor\sqrt{N_{h, c}}\right\rfloor$ nodes starting from node $\left(\left\lceil\sqrt{N_{h, c}}\right\rceil, 1\right)$, going toward node $\left(\left\lceil\sqrt{N_{h, c}}\right\rceil,\left\lceil\sqrt{N_{h, c}}\right\rceil\right)$ along row $\left\lceil\sqrt{N_{h, c}}\right\rceil$ and then eventually, if $N_{h, c}-\left\lfloor\sqrt{N_{h, c}}\right\rfloor^{2}>\left\lceil\sqrt{N_{h, c}}\right\rceil$, up along column $\left\lceil\sqrt{N_{h, c}}\right\rceil$ taking nodes $\left(\left\lceil\sqrt{N_{h, c}}\right\rceil-1,\left\lceil\sqrt{N_{h, c}}\right\rceil\right),\left(\left\lceil\sqrt{N_{h, c}}\right\rceil-2,\left\lceil\sqrt{N_{h, c}}\right\rceil\right)$, and so on.

Let the order of $G_{h}$ be $n_{h}=\left\lfloor\sqrt{N_{h, c}}\right\rfloor$, that is the number of rows or columns of the largest subgrid contained in $G_{h}$. We now show an incremental construction for layouts with edge congestion at most $c$ such that, for any positive integer $h$, the subgraph induced by all the nodes with hop count at most $h$ is $G_{h}$ (see Figure 1). The theorem then follows by considering the restriction of the layout on the $n_{h} \times n_{h}$ subgrid of $G_{h}$ containing $n_{h}^{2}=E(h, c)$ nodes.

Clearly $G_{0}$ contains only the root $(1,1)$ and a $\langle 1, c\rangle$-edge layout for $G_{1}$ can be easily constructed by putting a suitable $V P$ from the root to each node in $G_{1}$. Let us now show when $h \geq 1$ how to construct from a $\langle h, c\rangle$-edge layout for $G_{h}$ a $\langle h+1, c\rangle$-edge layout for $G_{h+1}$. Notice that, for any node $(i, j)$, all the nodes $\left(i^{\prime}, j^{\prime}\right)$ with $i^{\prime} \leq i$ and $j^{\prime} \leq j$ belong to a shortest path from $(i, j)$ to the root $(1,1)$. Then, we first have a set of expanding VPs that, for each row $i$ (resp. column $i$ ) with $1 \leq i \leq n_{h}$, are between the nodes in row $i$ (resp. column $i$ ) with hop count $h$ (that is belonging to $G_{h}$ but not to $G_{h-1}$ ) and the nodes in row $i$ (resp. column $i$ ) belonging to $G_{h+1}-G_{h}$, so that each of them is reached in $h+1$ hops. All the remaining available VPs from the nodes in $G_{h}-G_{h-1}$ are used to reach the remaining not considered nodes $(i, j)$ of $G_{h+1}-G_{h}$ with $i>n_{h}$ and $j>n_{h}$, i.e. in the right-down corner.

For any given row $i$ (resp. column $i$ ) with $1 \leq i \leq n_{h}$, let $d_{h}$ be the number of nodes in row $i$ (resp. column $i$ ) belonging to $G_{h+1}-G_{h}$. Since each edge can 


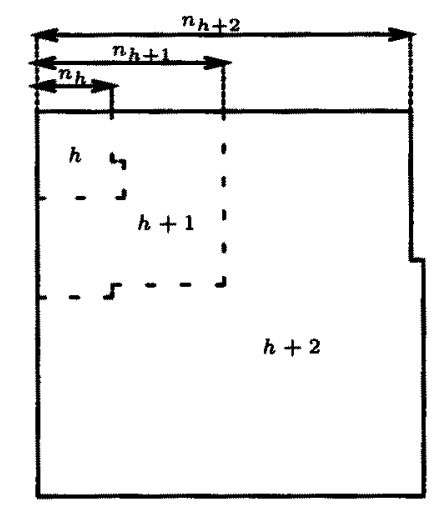

Fig. 1. The incremental layout for grids

have congestion at most $c$, in order to guarantee the correctness of the above incremental construction we have to prove that the $c \cdot d_{h} \geq d_{h+1}$.

By construction,

$$
\begin{gathered}
d_{h} \leq n_{h+1}-n_{h}+1=\left\lfloor\sqrt{N_{h+1, c}}\right\rfloor-\left\lfloor\sqrt{N_{h, c}}\right\rfloor+1 \leq \\
\sqrt{\frac{(2 c)^{h+2}-1}{2 c-1}}-\sqrt{\frac{(2 c)^{h+1}-1}{2 c-1}}+2= \\
\sqrt{\frac{(2 c)^{h+1}}{2 c-1}}\left(\sqrt{2 c-\frac{1}{(2 c)^{h+1}}}-\sqrt{1-\frac{1}{(2 c)^{h+1}}}\right)+2< \\
\sqrt{\frac{(2 c)^{h+1}}{2 c-1}}\left(\sqrt{2 c-\frac{1}{2 c}}-\sqrt{1-\frac{1}{2 c}}\right)+2= \\
\sqrt{\frac{(2 c)^{h+1}}{2 c-1}}\left(\sqrt{\frac{(2 c-1)(2 c+1)}{2 c}}-\sqrt{\frac{2 c-1}{2 c}}\right)+2= \\
\sqrt{(2 c)^{h+1}}\left(\frac{\sqrt{2 c+1}-1}{\sqrt{2 c}}\right)+2 .
\end{gathered}
$$

Similarly,

$$
\begin{gathered}
d_{h} \geq n_{h+1}-n_{h}-1=\left\lfloor\sqrt{N_{h+1, c}}\right\rfloor-\left\lfloor\sqrt{N_{h, c}}\right\rfloor-1 \geq \\
\sqrt{\frac{(2 c)^{h+2}-1}{2 c-1}}-\sqrt{\frac{(2 c)^{h+1}-1}{2 c-1}}-2>\sqrt{(2 c)^{h+1}}\left(\frac{\sqrt{2 c}-1}{\sqrt{2 c-1}}\right)-2 .
\end{gathered}
$$


It is possible to verify that if $4 \leq c \leq 11$ and $h \geq 2$ and if $c \geq 12$, any $h$ it is

$$
c d_{h}>c \sqrt{(2 c)^{h+1}}\left(\frac{\sqrt{2 c}-1}{\sqrt{2 c-1}}\right)-2 c \geq \sqrt{(2 c)^{h+2}}\left(\frac{\sqrt{2 c+1}-1}{\sqrt{2 c}}\right)+2>d_{h+1} .
$$

A case analysis shows that the construction works also for $4 \leq c \leq 11$ and $0 \leq h<2$. This completes the proof of the theorem.

Consider now a square torus $T_{n \times n}$ of $N=n^{2}$ nodes. Similarly to grids, for edge congestion $c=1$ we have the following theorem.

Theorem 25. Let $\mathcal{R}^{2}$ be the family of square torus graphs, then $E(h, 1)=$ $(2 h-1)^{2}$ if $h \leq 3$, otherwise $E(h, 1)=(2 h+1)^{2}$.

Theorem 26. Let $\mathcal{R}^{2}$ be the family of square torus graphs. Then, for $c \geq 4$, $E(h, c)=\left\lfloor\sqrt{N_{h, c}}\right\rfloor^{2}$, where $N_{h, c}=4\left(\frac{(2 c)^{h+1}-1}{2 c-1}-\frac{c^{h+1}-2 c^{2}+4 c}{c-1}\right)$.

Sketch of proof. Since tori are node-symmetric, we can consider any node as the root. Unfortunately, Lemma 9 does not to give an exact estimation of the number of nodes reachable in $h$ hops for any given $h$, since all the nodes in the same row or column of the root have parameter 3 , that is can exploit 3 edges to reach the other nodes, while all the remaining ones have parameter 2 .

A finer argument allows the exact estimation of $N_{h, c}$ in the clair. and then, after defining like for grids a similar notion of toroid, the proof is similar to that of Theorem 24. The complete proof of the theorem will appear in the full paper.

Tighter results can be determined for the node congestion case.

Theorem 27. Let $\mathcal{P}^{2}$ be the family of square grid graphs, then $N(h, c)=$ $\left\lfloor\sqrt{\frac{c(c-1)^{h}-2}{c-2}}\right\rfloor^{2}$.

Theorem 28. Let $\mathcal{R}^{2}$ be the family of square torus graphs, then $N(h, c)=$ $\left\lfloor\sqrt{\frac{c(c-1)^{h}-2}{c-2}}\right\rfloor^{2}$.

In the above two theorems the upper bound on $N(h, c)$ derives directly from Lemma 10, while matching layouts are constructed as in the edge congestion case by exploiting the gridoid and toroid methods. The formal proofs of these theorems will appear in the complete paper.

Before concluding the section, we remark that the gridoid and toroid methods can be easily extended to the $d$-dimensional case with $d>2$, thus yielding corresponding optimal edge or node layouts for $d$-dimensional grids an tori. Even if we don't put separate claims, for $c$ is suitably large in $d$-dimensional grids this yields $E(h, c)=\left\lfloor\left(\frac{(d c)^{h+1}-1}{d c-1}\right)^{\frac{1}{d}}\right\rfloor^{d}$ and $N(h, c)=\left\lfloor\left(\frac{c(c-1)^{h}-2}{c-2}\right)^{\frac{1}{d}}\right\rfloor^{d}$, while $N(h, c)=\left\lfloor\left(\frac{c(c-1)^{h}-2}{c-2}\right)^{\frac{1}{d}}\right\rfloor^{d}$ also for $d$-dimensional tori. 


\section{Conclusion and open problems}

In this paper we have considered two new cost measures related to the communication overhead and the space requirements associated to virtual path layouts in ATM networks, that is the edge congestion and the node congestion.

All the provided time complexity results are tight, and the same holds for the layout constructions for specific topologies, except for the cases of an edge congestion $c=2$ and $c=3$ in grids and tori. We are very close to the determination of these layouts, but they are not incremental, that is the subset of the nodes with hop count at most equal to a given integer $h$ in general does not form a gridoid or a toroid. In fact, it is possible to see that in these cases the incremental solution does not work, as there are values of $h$ such that from the gridoid or toroid of the nodes with hop count at most $h$ it is not possible to build the successive one corresponding to a hop count at most equal to $h+1$. Similar considerations hold for the $d$-dimensional cases with $d>2$.

An interesting issue to be pursued is the determination optimal path layouts for other network topologies. Moreover, it would be interesting to extend all these results to all-to-all layouts, where communication must be guaranteed between any two pair of nodes.

Another open question concerns the relationship between the congestion measures defined in this paper and the load parameter, that is the number of VPs that share a physical edge. For instance, as it can be easily verified, a load $l$ implies an edge congestion $c \leq l$, but apart from this case it seems that there is no strong relationship between the different parameters.

Finally, while we have remarked that in this context it does not make sense to consider an unbounded stretch factor, a case worth to investigate is when the stretch factor is bounded by a given real number greater than one.

\section{References}

1. S. Ahn, R.P. Tsang, S.R. Tong, and D.H.C. Du. Virtual path layout design on ATM networks. In INFOCOM'94, pages 192-200, 1994.

2. J. Burgin and D. Dorman. Broadband ISDN resource management: The role of virtual paths. IEEE Communicatons Magazine, 29, 1991.

3. I. Cidon, O. Gerstel, and S. Zaks. A scalable approach to routing in ATM networks. In G. Tel and P.M.B. Vitányi, editors, 8th International Workshop on Distributed Algorithms (LNCS 857), pages 209-222, Terschelling, The Netherlands, October 1994. Submitted for publication in IEEE/ACM Trans. on Networking.

4. R. Cohen and A. Segall. Connection management and rerouting in ATM networks. In INFOCOM'94, pages 184-191, 1994.

5. T. Eilam, M. Flammini, and S. Zaks. A complete characterization of the path layout construction problem for ATM networks with given hop count and load. To appear in the Proc. of the 24th International Colloquium on Automata, Languages and Programming (ICALP), 1997.

6. M.R. Garey and D.S. Johnson. Computers and Intractability: A Guide to the Theory of NP-Completeness. W.H. Freeman and Co., 1979. 
7. O. Gerstel, I. Cidon, and S. Zaks. The layout of virtual paths in ATM networks. IEEE/ACM Transactions on Networking, 4(6):873-884, 1996.

8. O. Gerstel, A. Wool, and S. Zaks. Optimal layouts on a chain ATM network. In 3rd Annual European Symposium on Algorithms (ESA), (LNCS 979), Corfu, Greece, September 1995, pages 508-522. To appear in Discrete Applied Mathematics.

9. O. Gerstel and S. Zaks. The virtual path layout problem in fast networks. In 13th ACM Symp. on Principles of Distributed Computing, pages 235-243, Los Angeles, USA, August 1994.

10. R. Händler and M.N. Huber. Integrated Broadband Networks: an introduction to ATM-based networks. Addison-Wesley, 1991.

11. ITU recommendation. I series (B-ISDN), Blue Book, November 1990.

12. F.Y.S. Lin and K.T. Cheng. Virtual path assignment and virtual circuit routing in ATM networks. In GLOBCOM'93, pages 436-441, 1993.

13. C. Partridge. Gigabit Networking. Addison Wesley, 1994.

14. K.I. Sato, S. Ohta, and I. Tokizawa. Broad-band ATM network architecture based on virtual paths. IEEE Transactions on Communications, 38(8):1212-1222, August 1990.

15. Y. Sato and K.I. Sato. Virtual path and link capacity design for ATM networks. IEEE Journal of Selected Areas in Communications, 9, 1991. 